\title{
Virtual laboratories in (bio)chemical engineering education
}

\author{
Lucília Domingues*, Isabel Rocha, Fernando Dourado, \\ Madalena Alves, Eugénio C. Ferreira
}

IBB-Institute for Biotechnology and Bioengineering, Centre of Biological Engineering, Universidade do Minho, Campus de Gualtar, 4710-057 Braga, Portugal

\begin{abstract}
A B S T R A C T
In the last decades, Information and Communications Technologies (ICT) have been promoting the creation and adoption of new learning and teaching styles. Virtual laboratories, by overcoming some limitations of conventional hands-on experiments, have been adopted as a complement or in substitution of laboratory sessions.

This paper describes the design and implementation of two virtual labs for biochemical engineering education intended for students at the BSc degree.

One of the virtual labs is intended to fully replace the hands-on experiment and consists on the determination of the correlation between oxygen transfer rate, aeration rate and agitation power in a reactor. The other virtual lab consists on the determination of the residence time distribution (RTD) in continuous stirred tanks series and was implemented to support the physical experiments rather than replacing them.

The virtual labs provide the students a learning platform covering the fundamentals underlying the experiment, its pre-visualization and simulation. The effectiveness of the implemented system was evaluated through direct experimentation and survey (through questionnaires) with students taking the chemical technology lab course. For the RTD virtual Lab, and based on specific learning outcomes, teachers could assess significant improvement in students' performance in the lab and also a more thorough discussion of the results in the reports. The survey results show that, in average, considering the two virtual labs and several classes, $93 \%$ of the students consider the virtual labs of great utility.
\end{abstract}

(c) 2010 The Institution of Chemical Engineers. Published by Elsevier B.V. All rights reserved.

Keywords: Virtual labs; e-Learning; (Bio)chemical engineering education

\section{Introduction}

Information and Communications Technologies (ICT) facilitates the development of novel teaching strategies for laboratory classes, including new approaches to illustration, simulation, demonstration, experimentation, operation, and communication.

While the "hands-on" approach for laboratory experiments has enormous educational value, these traditional teaching methods are expensive and require complex logistics regarding space, staff, scheduling and safety (Feisel and Rosa, 2005; Selmer et al., 2007). Virtual laboratories (herein "virtual labs") may allow overcoming these limitations by allowing a computerized simulation of the laboratory experiments (Rasteiro et al., 2009). Even though virtual labs cannot fully substitute the hands-on laboratory experiments in engineering curricula, they also provide several advantages as a complementary educational tool, the most notorious being the possibility of performing them anytime at any place provided Internet access is available (Ferreira, 2004). Virtual labs have been considered as a support to physical laboratories (Rafael et al., 2007; Shin et al., 2002) and even remote laboratories may be used as a complement to lab sessions (Klein and Wozny, 2006).

The University of Minho has been promoting a pilot project that concerns the development of components and software agents for assistance in laboratory lessons of curricular units from Science and Technology areas-the VLabs Project. These software contents will lead to the establishment of virtual laboratories and are expected to complement the students' lab work, as well as to promote new e-Learning education activ-

\footnotetext{
* Corresponding author. Tel.: +351 253 604400; fax: +351 253678986.

E-mail address: luciliad@deb.uminho.pt (L. Domingues).

Received 3 April 2009; Received in revised form 11 November 2009; Accepted 4 February 2010

1749-7728/\$ - see front matter (c) 2010 The Institution of Chemical Engineers. Published by Elsevier B.V. All rights reserved. doi:10.1016/j.ece.2010.02.001
} 


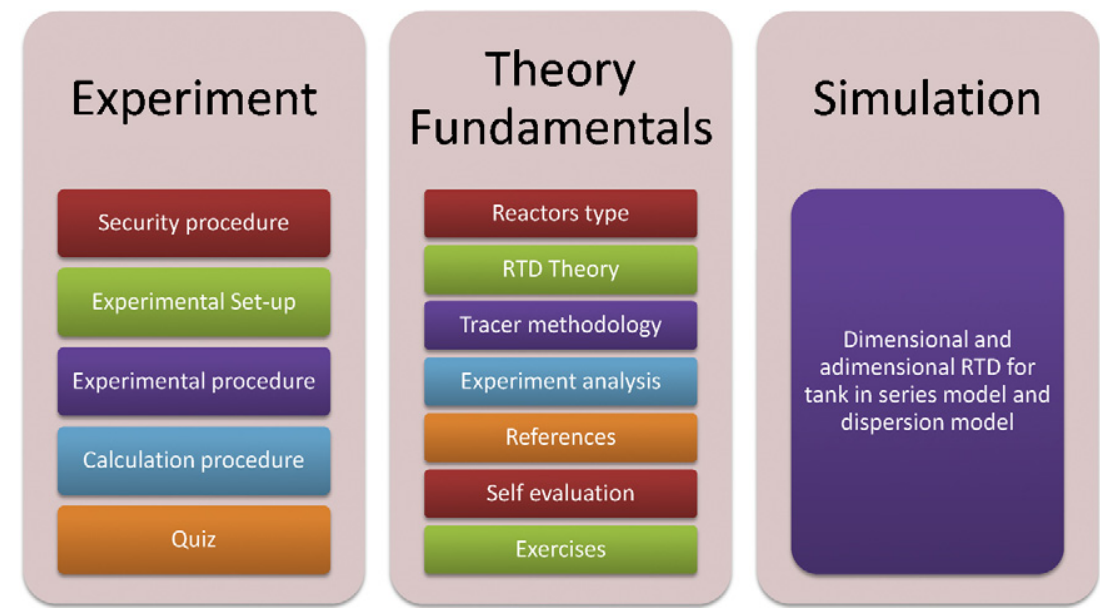

Fig. 1 - Web platform structure of the "Determination of the Residence Time Distribution in Continuous Tank Series" virtual lab.

ities. The portal http://vlabs.uminho.pt allows access to the virtual laboratories where animations, videos and control of virtual experiments can be handled, providing extra digital content for laboratory classes and for curricular units without an assigned laboratory schedule. Contents and didactic material are currently being developed for the virtual laboratories, in which the students can explore details on various laboratory-scale equipments like microscopes and reactors, as well as come in contact with intangible realities like the mechanisms behind catalytic reactions.

The Biological Engineering Department is engaged in this VLabs Project. The adoption of these tools in teaching activities and their offering over the Internet will permit a better preparation of the students before attending the laboratory classes or the simulation and observation of certain keyexperiments.

This paper describes the design and implementation of two virtual labs for biochemical engineering education:

\footnotetext{
- "Determination of the Residence Time Distribution in Continuous Tank Series-RTD";

- "Determination of the Correlation between oxygen transfer rate, aeration rate and agitation power-A\&A".
}

These virtual labs were designed for the course "Chemical Technology Laboratory" (CTL), taught to the third year students of the Biological Engineering five-years integrated Master degree and were first implemented in 2006. The two virtual labs have distinct goals: while A\&A aims at replacing the hands-on experiment, RTD will support the hands-on experiment.

\section{Implementation and results}

\subsection{Determination of the residence time distribution (RTD) in continuous tank series}

\subsubsection{Experiment description}

This experiment aims at the experimental determination of the residence time distribution in a cascade of $1,2,3$, and 4 stirred tanks (see for example Fogler, 1986; Levenspiel, 1972 for the fundamentals of this work) and the application of the "Tanks in Series Model" to a series of continuous stirred tank reactors. Students are also encouraged to apply the "Dispersion Model" and to use the Solver feature of the Excel ${ }^{\circledR}$ spreadsheet (Ferreira et al., 2004) to determine the model parameters-the Peclet number (for the dispersion model) and the number of tanks (for the tanks in series model) from the best fits of their experimental data. The experimental RTD is obtained using methylene blue as tracer. After a pulse injection, its concentration profile is followed at the tanks' outlet by spectrophotometry. This experiment has been implemented in the CTL course for many years, as a traditional lab experiment. It was adapted for the VLabs because, even though it is very easy and safe to perform in the laboratory, the students' written reports generally showed a insufficient knowledge of the RTD concepts, as well as strong limitations in the discussion of the results obtained from their experiments.

\subsubsection{Virtual lab goals and gains}

The main goal with this virtual lab was to provide a web support to the traditional lab experiment, thus improving student's effectiveness and autonomy in the laboratory class, in data analysis and report writing.

Even though this virtual lab was not designed to replace the hands-on experiment, it reduced costs as it allowed decreasing the time needed to conduct the experiment, decreasing the number of repetitions (due to errors), and also eliminating the need for full-time supervision.

\subsubsection{Web platform description}

The web platform structure is presented in Fig. 1 and is divided into three main sections: theory fundamentals, experiment and simulation.

In the theory fundamentals section, the theory underlying the experiment is presented with animations and illustrations. Also, a self-evaluation test can be done and some exercises are proposed.

In the experiment section, the safety procedures that students must learn and practice once in the lab are given. Also, a detailed description of the experimental set-up is provided through an interactive platform, where the students can view details on specific equipment just by placing the computer mouse on top of the corresponding picture. To enhance the visual experience, parts of the experimental procedure are detailed in videos that show some relevant aspects of the experimental assay, accompanied by written text and sound, explaining what is being executed. In this section, the experimental protocol and a template for lab data registration are also available for download, so that students can take it to the 

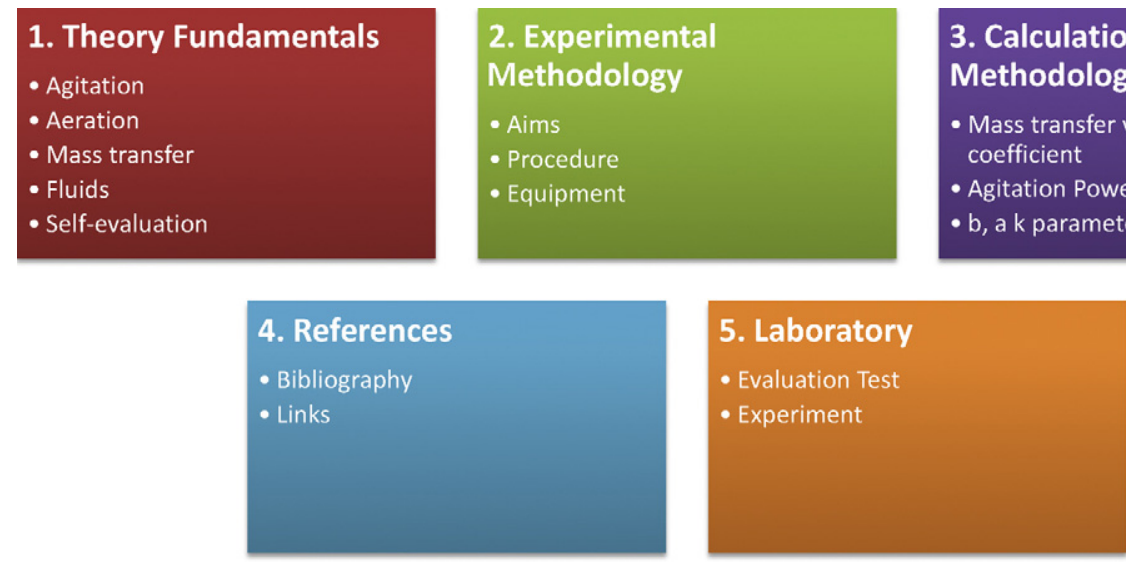

Fig. 2 - Web platform structure of the "Determination of the correlation between oxygen transfer rate, aeration rate and agitation power" virtual lab.

lab. Also, there is a calculation procedure implemented in a questionnaire format so that the students can think by themselves before the answer is provided by the software. Finally, a test-quiz has to be answered by the students. This test is meant to evaluate if the students are prepared to do the laboratory experiment. Once the students complete the test, the results are sent automatically to the teacher by email. If a score above the $50 \%$ threshold is obtained, the student is authorized to do the experiment.

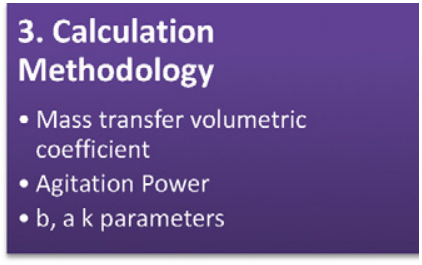

3. Calculation

Mass transfer volumetric

coefficient

Agitation Power

b, a k parameters
In the simulation section the students can perform RTD experiments using either the tanks in series model or the dispersion model. They can set the volumetric flow rate, the tank volume and model parameters (the number of tanks for the tanks in series model and the Peclet number for the dispersion model). Students can also use the simulation section to determine the most suitable time interval for sample collection, a relevant parameter for performing the RTD laboratory experiments.

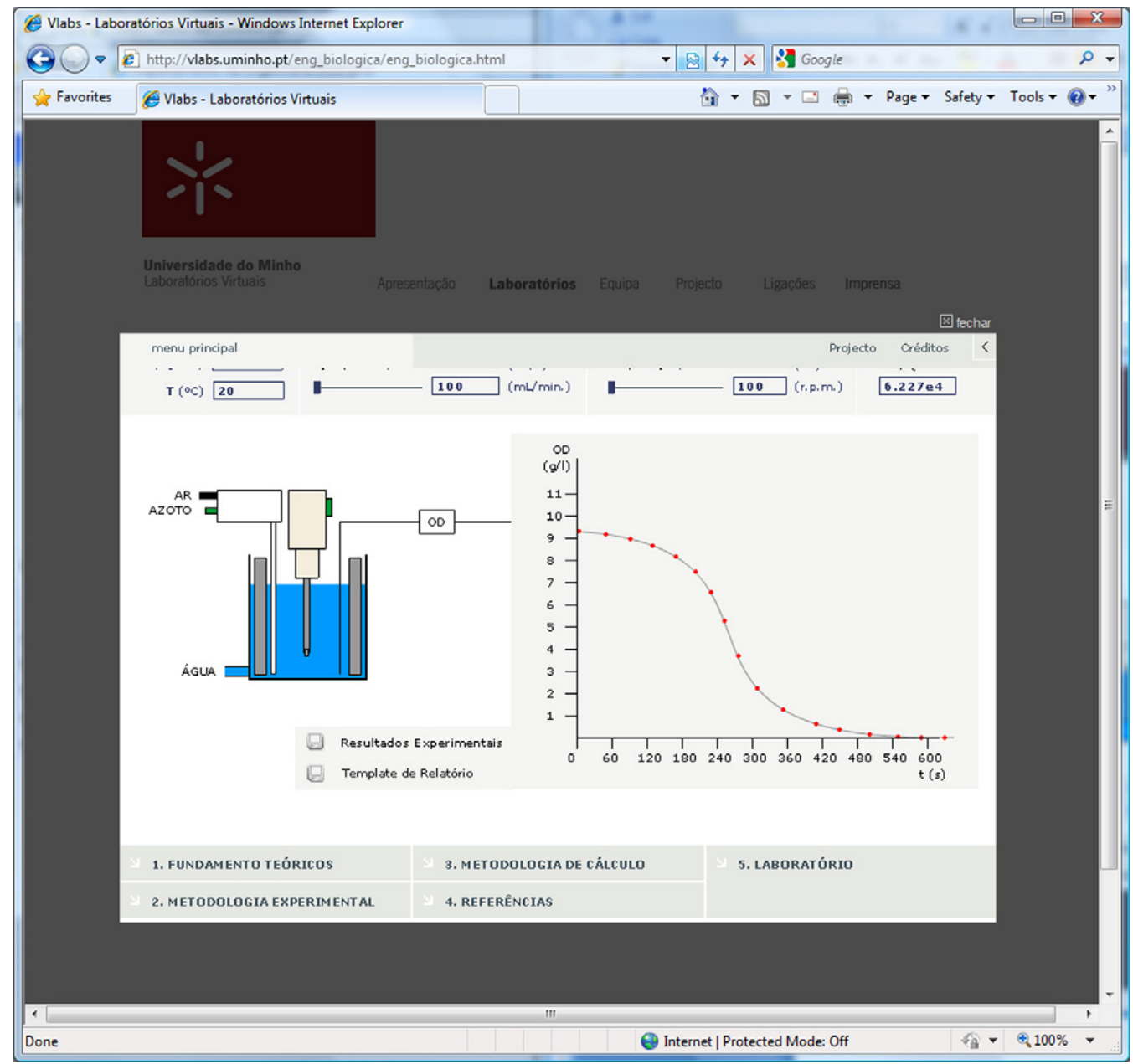

Fig. 3 - Experiment platform for the "Determination of the correlation between oxygen transfer rate, aeration rate and agitation power" virtual lab. 


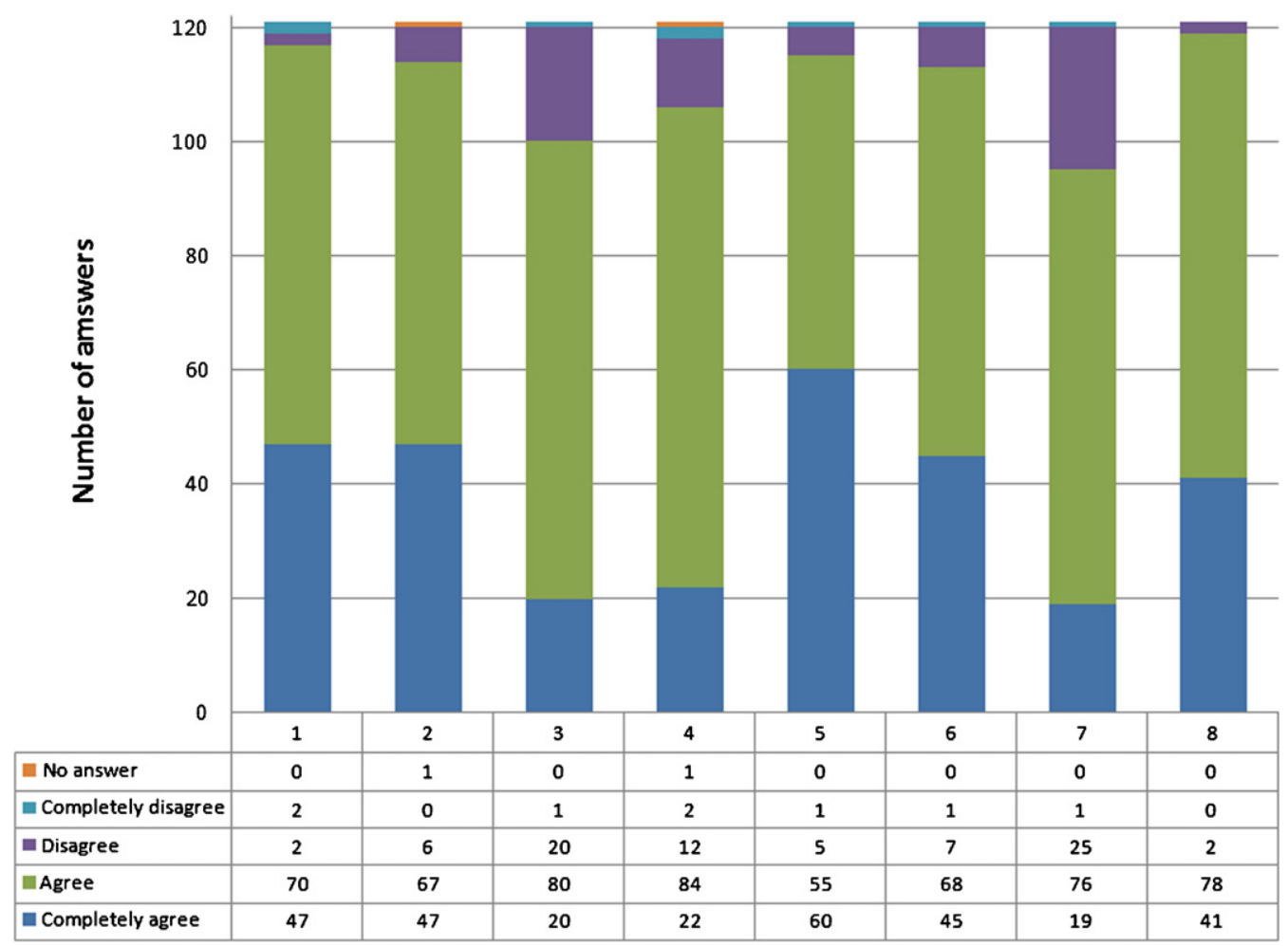

Fig. 4 - Students' survey results for the RTD virtual lab (2006/2007; 2007/2008 and 2008/2009 results). Question 1: The RTD virtual Lab contents have led to a better understanding of the concepts necessary for the execution of the experimental work; 2: the RTD virtual Lab contents have made the execution of the experimental work easier; 3: the RTD virtual Lab contents have made the calculation procedure easier; 4: the RTD virtual Lab contents have helped in the analysis and interpretation of the results; 5: the RTD virtual Lab portal is easy to use?; 6: the RTD virtual Lab portal is well organized; 7: the RTD virtual Lab portal is complete; 8: in general, I consider the RTD virtual Lab portal of great utility.

2.2. Determination of correlations between oxygen transfer rate, aeration rate and agitation power (A\&A)

\subsubsection{Experiment description}

The focus of this virtual lab is on the oxygen transfer from air to liquid media and on the influence of aeration rate and stirring power on the oxygen transfer coefficient (the $k_{\mathrm{L}}$ a coefficient) (see for example Geankoplis, 1983; Wang et al., 1979 for the fundamentals of this work). This experiment had been implemented in the CTL course for some years as a traditional lab experiment. It consists in measuring the dissolved oxygen concentration after sparging water in a stirred tank with nitrogen with the subsequent introduction of air. Different aeration rates and stirrer speeds are tested in order to correlate those variables with the oxygen transfer coefficient.

This virtual lab was developed due to the high operational costs with nitrogen, and is intended to completely replace the hands-on experiment. This in fact has other advantages, since the experimental procedure although simple, is repetitive and monotonous.

\subsubsection{Virtual lab goals and gains}

In summary, the main goal of this implementation was to replace the hands-on experiment, allowing to save time and costs, while improving the students' education (this includes developing self-study, team work, initiative and organizational skills, to name a few). As a note, before this virtual lab implementation, the laboratory classes would last $6 \mathrm{~h}$. Before the classes, each work-group was required to meet with the teacher for a $15 \mathrm{~min}$ oral evaluation and discussion on the protocol, safety and waste disposal procedures. Now, $20 \mathrm{~min}$ are more than adequate to perform the simulations using any computer with an Internet connection.

\subsubsection{Web platform description}

The web platform structure, presented on Fig. 2, is divided into five different sections: theory fundamentals, experimental methodology, calculation methodology, references and laboratory.

The first three sections were designed to support the experiment, results analysis and report writing. In the first section, theory fundamentals are provided while in the second section, a brief description of the aims, procedure and equipment is presented. The detailed experimental protocol is available for download from the procedure section. The third section describes the calculation methodology to be applied to the gathered data.

The fifth section (Laboratory) is the section where the actual virtual experiment can be executed. It is divided into evaluation test and experiment. The students are only authorized to perform the experiment if they obtain more than $50 \%$ in an evaluation test. This test is generated automatically by the computer from a pool of questions stored in a database.

In the experiment platform, one can choose the air volumetric flow rate and the stirring speed for each experiment. Students have to conduct the same procedure as they would in a conventional lab experiment that is, to first turn on the water supply and fill the tank, and then start the stirrer. They should turn on the nitrogen supply so that the dissolved oxygen concentration drops to zero and then turn on the air supply. Fig. 3 shows the experiment platform. The left hand side is where the students can do the manipulations of the process 




Fig. 5 - Students survey results in aeration and agitation virtual lab (2006/2007 results). Question 1: The A\&A virtual Lab contents have led to a better understanding of the concepts necessary for the execution of the experimental work; 2: the virtual experiment replaces the hands-on experiment completely, there is no need to do the experiment on the lab.; 3: the A\&A virtual Lab contents have made the calculation procedure easier; 4: the A\&A virtual Lab contents have helped in the analysis and interpretation of the results; 5: the A\&A virtual Lab portal is easy to use; 6: the A\&A virtual Lab portal is well organized; 7: the A\&A virtual Lab portal is complete; 8: in general, I consider the A\&A virtual Lab portal of great utility.

(turn on/off water, nitrogen, air supplies and stirrer). On the right-hand side a real-time graphical illustration of dissolved oxygen versus time is shown. A new experiment with a different air flow rate can only be performed after the dissolved oxygen concentration had dropped to zero (after turning on again the nitrogen supply). The results can be downloaded in an Excel ${ }^{\circledR}$ worksheet and a report template is also available for download.

\section{Assessment/students feedback}

Both virtual labs (RTD and A\&A) were first implemented in the CTL course in 2005/2006. In this first year implementation of the virtual labs platform, instructors could assess the improvement of student's performance at three levels: (i) preparation of laboratory session assessed through a written questionnaire; (ii) organization and experimental skills at the laboratory session; and (iii) results interpretation analysis and discussion. These evaluations were quantitatively compared with the learning outcomes obtained in the previous five years.

In terms of preparation of laboratory sessions, a comparison between the former and the actual method is not straightforward. The former method was based on oral examinations in the laboratory class, before starting the session, whereas now the students have to answer a questionnaire on the web. The fundamental difference is the actual possibility of accessing all kind of information sources while answering the questionnaire. Considering the inherent advantages and disadvantages of both approaches, it is still not evident which one is better for learning purposes.
In terms of organization and skills at the laboratory session, it was possible to assess an increase from 60 to $90 \%$ in the number of students that attained the following learning outcome: "to plan and execute an experiment applying Chemical Reaction Engineering Concepts of RTD". This evaluation was based on the comparison of the average marks obtained by the students during the laboratory session, before (average of five years) and after the virtual lab implementation. The advantages of using virtual labs as preparation tools for laboratory sessions have also been noticed by others (Martinez-Jimenez et al., 2003; Woodfield et al., 2005; Dalgarno et al., 2009). Concerning the results interpretation analysis and discussion, it was possible to quantify an increase from 55 to $80 \%$ in the number of students that attained the following learning outcome: "to interpret, analyze and discuss experimental results in a quantitative way". This evaluation was based on the comparison of the average marks obtained by the students on the discussion section of the written report, before (average of five years) and after the virtual labs implementation. In the first year of implementation (2005/2006), students feedback was very positive but no assessment was made. Students perception was evaluated by a survey (through questionnaires) performed with students taking the CTL course in 2006/2007, and 2007/2008 and 2008/2009 for the RTD virtual lab and in 2006/2007 for the A\&A virtual lab. Due to Bologna implementation in the curriculum reform, this virtual lab has not been used in CTL classes after 2006/2007.

Data from the survey performed in 2006/2007, 2007/2008 and 2008/2009 for the RTD virtual lab, in a total of 121 students, are presented in Fig. 4. 
The students have considered that the virtual lab portal is easy to use (95\% agreed or completely agreed) and well organized (93\% agreed or completely agreed), and $79 \%$ have considered that it is complete. The majority of the students have agreed that the RTD virtual Lab contents led to a better understanding of the concepts necessary for the execution of the experimental work (97\%) and that it made easier the execution of the experimental work (94\%). They have also considered $(88 \%)$ that the virtual lab portal helped in the analysis and interpretation of the results. Only $83 \%$ have agreed that the RTD virtual lab has made the calculation procedure easier. Overall, $98 \%$ of the students have considered the virtual lab portal of great utility.

The students' survey results obtained for the aeration and agitation virtual lab, in a total of 30 students, are presented in Fig. 5.

Again, students have considered that the virtual lab portal is easy to use (97\%) and well organized (90\%). Interestingly, a higher number of responders disagreed or completely disagreed $(44 \%)$ when it was stated that the virtual experiment replaced the hands-on experiment completely. Nevertheless, one can see that opinions are quite positive towards the virtual lab portal.

\section{Summary and conclusions}

This paper describes the design and implementation of two virtual labs for biochemical engineering education. These virtual labs were designed for the course "Chemical Technology Laboratory" taught to the third year students of the Biological Engineering integrated Master studies in Minho University, Portugal. The experiment was first implemented in 2006.

One of the virtual labs consists on the determination of the correlation between oxygen transfer rate, aeration rate and agitation power in a reactor. This virtual lab is intended to fully replace the hands-on experiment. The advantages over performing the experience are clear: reduced costs, reduced experience time, and improved data with no loss of education efficiency. The other virtual lab consists on the determination of the residence time distribution (RTD) in continuous tanks series and was implemented to support the experiments rather than replacing them.

These virtual labs demonstrate that, by way of the Internet, there are remarkable tools to support the conventional hands-on laboratory experiments as they provide the students an experimental platform gathering the fundamentals underlying the experiment, its pre-visualization (videos, simulation) and data generation. This web support will improve the students' effectiveness and autonomy in the laboratory class, as well as in the analysis of the results and report writing.

The effectiveness of the implemented system was evaluated through direct experimentation and survey (through questionnaires) with students taking the chemical technology lab course. Teachers have noticed a clear improvement in students' performance in the lab and also a more thorough discussion of the results in the reports (for the RTD virtual lab). The survey results show that the majority of the students (93\% of a total of 151 students) consider the virtual labs portal of great utility.

\section{Acknowledgments}

This project is being supported by strategic funds from "Programa Qualidade" of the University of Minho. The students Cristiana Mota and Helena Venda are acknowledged for their contribution in the design of the A\&A virtual lab platform. Doctor Anthony Danko is acknowledged for English proofreading of the manuscript.

\section{References}

Dalgarno, B., Bishop, A.G., Adlong, W. and Bedgood, D.R., Jr., 2009, Effectiveness of a virtual laboratory as a preparatory for distance education chemistry students. Computers \& Education, 53: 853-865.

Feisel, L.D. and Rosa, A.J., 2005, The role of laboratory in undergraduate engineering education. Journal of Engineering Education, 94(1): 121-130.

Ferreira, E.C., 2004, Blended learning in bioprocess systems engineering education: issues, methods, and applications, In ESCAPE-14: European Symposium on Computer Aided Process Engineering Lisboa, May 16-19, (p. 6).

Ferreira, E.C., Lima, R. and Salcedo, R., 2004, Spreadsheets in chemical engineering education- $\mathrm{a}$ tool in process design and integration. International Journal of Engineering Education, 20(6): 928-938.

Fogler, H.S., (1986). Elements of Chemical Reaction Engineering. (Prentice-Hall International) (chapter 13)

Geankoplis, C.J., (1983). Transport Processes and Unit Operations. (Allyn and Bacon).

Klein, A. and Wozny, G., 2006, Web based remote experiments for chemical engineering education-The online distillation column. Transactions of IChemE, Part D, Education for Chemical Engineers, 1: 134-138.

Levenspiel, O., (1972). Chemical Reaction Engineering. (Wiley International Edition) (chapter 9)

Martinez-Jimenez, P., Pontes-Pedrajas, A., Polo, J. and Climent-Bellido, M.S., 2003, Learning in chemistry with virtual laboratories. Journal of Chemical Education, 80(3): 346-352.

Rafael, A.C., Bernardo, F., Ferreira, L.M., Rasteiro, M.G. and Teixeira, J.C., 2007, Virtual applications using a web platform to teach chemical engineering: the distillation case. Transactions of IChemE, Part D, Education for Chemical Engineers, 2(1): 20-28.

Rasteiro, M.G., Ferreira, L., Teixeira, J., Bernardo, F.P., Carvalho M.G., Ferreira, A., Ferreira, R.Q., Garcia, F., Baptista, C.M.S.G., Oliveira, N., Quina, M., Santos, L., Saraiva, P.A., Mendes, A., Magalhães, F., Almeida, A.S., Granjo, J., Ascenso, M., Bastos, R.M. and Borges, R., 2009, LABVIRTUAL-a virtual platform to teach chemical processes. Education for Chemical Engineers, 4: e9-e19.

Selmer, A., Kraft, M., Moros, R. and Colton, C.K., 2007, Weblabs in chemical engineering education. Transactions of IChemE, Part D, Education for Chemical Engineers, 2: 38-45.

Shin, D., Yoon, E.S., Lee, K.Y. and Lee, E.S., 2002, A web-based, interactive virtual laboratories system for unit operations and process systems engineering education: issues, design and implementation. Computers and Chemical Engineering, 26: 319-330.

Wang, D., Cooney, C.L., Demain, A.L., Dunnill, P., Humphrey, A.E. and Lilly, M.D., (1979). Fermentation and Enzyme Technology. (John Wiley \& Sons, New York).

Woodfield, B.F., Andrus, M.B., Waddoups, G.L., Moore, M.S., Swan, R., Allen, R., Bodily, G., Andersen, T., Miller, J., Simmons, B. and Stanger, R., 2005, The virtual chem lab project: a realistic and sophisticated simulation of organic synthesis and organic qualitative analysis. Journal of Chemical Education, 82(11): 1728-1735. 